Journal of Case Reports 2019;9(2):92-94

\title{
Incarcerated Vaginal Pessary
}

\author{
Anubhuti Patel, Sweta Singh, Mitali Mahapatra \\ Department of Obstetrics \& Gynaecology, All India Institute of Medical Sciences, Bhubaneswar, Odisha, India.
}

\section{Corresponding Author:}

Dr. Anubhuti Patel

Email: dr.anubhuti.jbp@gmail.com

This is an Open Access article distributed under the terms of the Creative Commons Attribution License (creativecommons.org/ licenses/by/3.0).

Received Accepted

Published

November 26, 2018

April 21, 2019

April 30, 2019

\begin{abstract}
Background: Women on vaginal pessary required regular follow-up and good pessary care to avoid complications such as infection, ulceration and incarceration and rare complication like recto-vaginal or vesico-vaginal fistula. We present a case of a neglected vaginal ring pessary which got entrapped or embedded in a band of vaginal tissue. Case Report: A 53 year-old post-menopausal woman presented with bleeding per vaginum. She had history of insertion of a silicone ring-shaped vaginal pessary for the management of uterine prolapse one year back. The patient neglected the pessary, did not turn for follow-up. Pessary had been incarcerated in the vaginal wall; which was impossible to remove. She had excision of band of vaginal tissue and removal of the pessary under general anaesthesia. Conclusion: Women on vaginal pessary need regular follow-up to avoid complications such as infection, ulceration and incarceration.
\end{abstract}

Keywords: Pessaries, Pelvic Organ Prolapse, Postmenopause, Pregnancy, Uterine Prolapse.

\section{Introduction}

Pelvic organ prolapse is a common gynaecological condition that causes poor quality of life of affected women. $50 \%$ of multiparous women may be affected with this condition [1]. Management options are pelvic floor exercises, pessary insertion or surgical correction (pelvic floor repair) [2]. Over the period of time, the use of vaginal pessary has been shown to be an effective, safe, and highly acceptable mode of management in this condition [3]. It has a high success rate, high patient satisfaction and is associated with minimal complication when patient is in regular follow up and taking care of the pessary on her own [3]. It is cost-effective in the treatment of pelvic organ prolapse [4]. It is recommended as the first-line treatment for all women presenting with uterine prolapse and stress incontinence [3].

Common complications of pessary use are vaginal irritation, allergic reactions, leukorrhea, bleeding and voiding and defecating dysfunction.
Neglected pessary if left in situ for many years, may erode into the mucosa of rectum or bladder causing rectovaginal or vesicovaginal fistula $[5,6]$. Use of vaginal pessary is a safe option for conservative management but regular follow up and patient self pessary care is necessary to avoid complications.

\section{Case Report}

A 53 year old para 3 live 3, post-menopausal women presented to gynaecology out-patient department with complaints of vaginal bleeding. She had history of uterine prolapse for two years for which she was treated with vaginal ring pessary one year back. Her symptoms were relieved and she did not turn for follow up because she had no complains and did not adhere to the instruction for care of the vaginal pessary. For the symptom of vaginal bleeding she visited a private clinic. The gynaecologist had attempted to remove the incarcerated pessary without success and referred to tertiary health centre. 
Speculum examination of the vagina showed displaced vaginal pessary embedded in the posterior-lateral wall of vaginal with a $2 \mathrm{~cm}$ band of granulation tissue of vaginal epithelium overgrowing it, with fresh bleeding. A digital vaginal examination revealed a ring pessary stuck to the vagina and embedded in the postero-lateral vaginal wall. On per rectal examination rectal mucosa was found not to be involved.

Patient and relatives were counseled for surgical removal. Baseline investigations such as haematocrit and urinalysis were carried out and the results were within normal limits. Removal of the ring pessary was done by excision of the bands of vaginal tissue under general anaesthesia. Per rectal examination was normal. Vaginal packing was done and the patient was monitored for vaginal bleeding after the procedure. Post-operative period was uneventful. She was given antibiotics and analgesics and was discharged the next day. On follow up after 2 weeks she was asymptomatic.

\section{Discussion}

Pelvic floor repair by surgical correction is the definitive treatment of pelvic organ prolapse [7]. The use of vaginal pessary is an acceptable and satisfactory treatment option for conservative management in those women who are unfit for surgery due to co-morbid conditions, not willing for surgery, pregnant or wants to have more children [8]. They are safe, easy to insert but require self care by patient or by care taker or by health care provider. Since elderly women may also suffer from forgetfulness and dementia, neglected pessary care may cause complication like vaginal discharge, allergic reaction, vaginal irritation and erosion. Forgotten pessary may cause pressure necrosis and ulcerative mucosa which may later heal over the rim of pessary leading to impaction. These complications can be prevented by ensuring appropriate fitting, local oestrogen use and regular follow-up [9].
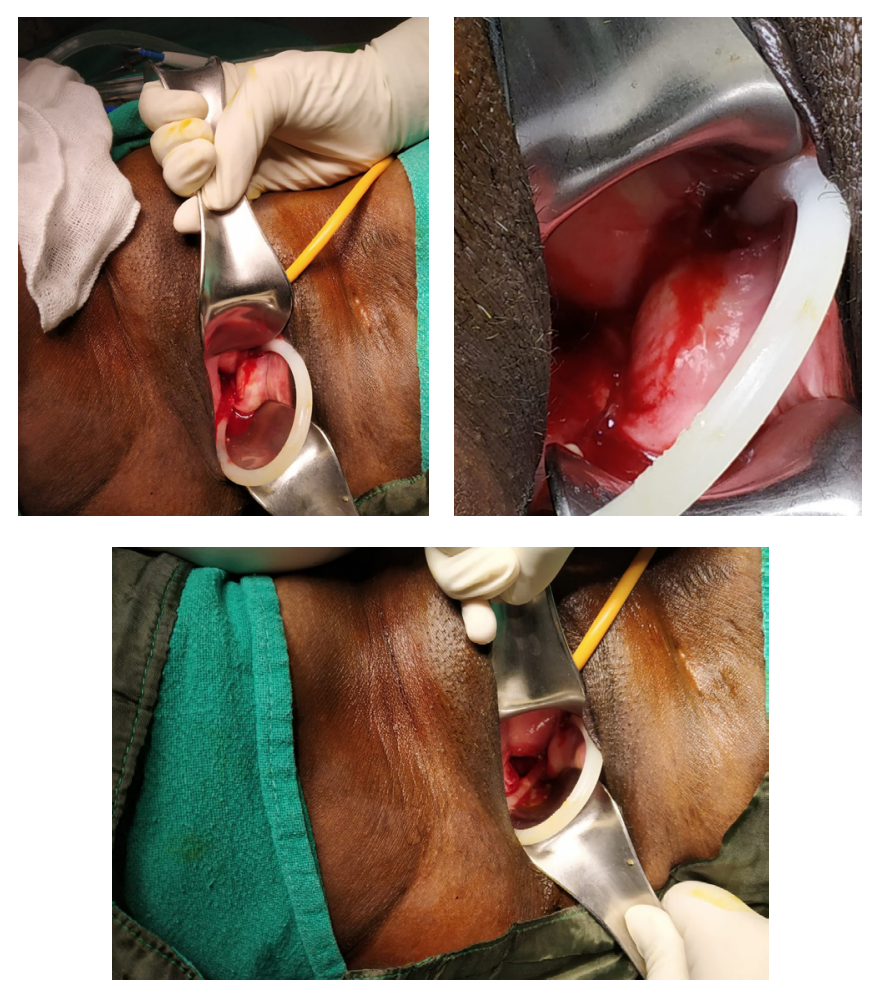

Fig.1-3: Incarcerated vaginal pessary.

Local application of estrogen cream reduces inflammation, allow easy removal of embedded pessary and help in epithelisation of vaginal mucosa. Surgical removal is required in rare cases with deeply embedded pessary. After insertion of pessary, patient and care taker should be instructed and educated for need for regular follow up. Initial follow up in the first two weeks is required to assess the fitness and whether patient is compliant with self-care protocols [3]. Pessary should be removed weekly, properly cleaned with soap and water and re-inserted. Speculum examination should be done in follow up visits. Vagina should be inspected for any newly developed erosion or ulceration which may be due to pressure effect. In these cases, pessary should be removed for 2-3 weeks and estrogen cream should be applied. Pessary of a different size should be inserted on healing of the ulcer/erosion. The pessary should be examined for any cracking, discoloration and deformation, and replaced as needed along with regular follow up intervals $[9,10]$. 


\section{Conclusion}

Complications of pessary insertion are usually associated with neglect of device following prolonged use. The patient should be trained for insertion, regular removal, cleaning and need for strictly timed follow-up visits.

Contributors: AP: manuscript writing, patient management; SS: manuscript editing, patient management; MM: critical inputs into the manuscript and patient management. AP will act as guarantor. All authors approved the final version of this manuscript.

Funding: None; Competing interests: None stated.

\section{References}

1. Swift SE. The distribution of pelvic organ support in a population of female subjects seen for routine gynecologic health care. Am J Obstet Gynecol. 2000;183:277-285.

2. Sivasuriya M. Cervical entrapment of a polythene vaginal ring pessary a clinical curiosity. Aust N Z J Obstet Gynaecol. 1987;27(2):168-169.
3. Robert M, Schulz JA, Harvey M. Technical update on pessary use. J Obstet Gynaecol Can. 2013;35:664-674.

4. Hullfish KL, Trowbridge ER, Stukenborg GJ. Treatment strategies for pelvic organ prolapse: a cost-effectiveness analysis. Int Urogynecol J Pelvic Floor Dysfunct. 2011;22:507-515.

5. Scotti RJ, Vargas I, Lippman L. Perforation and fistulization from a vaginal ring pessary. J Gynecol Surg. 1994;10:93-96.

6. Hanavadi S, Durham-Hall A, Oke T. Forgotten vaginal pessary eroding into the rectum. Ann R Coll Engl. 2004;86:1-2.

7. Rosenblum N, Pelvic organ prolapse: Considerations in surgical management: NYU Case of the Month, April 2017. Rev Urol. 2017;19(2):134-137.

8. Zeitlin MP, Lebher TB. Pessaries in the geriatric patient. J Am Geriatr Soc. 1992;40:635-639.

9. $\mathrm{Wu}$ V, Farrell SA, Baskett TF, Flowerdew G. A simplified protocol for pessary management. Obstet Gynecol. 1997;90:990-994.

10. Jones KA, Harmanli O. Pessary use in pelvic organ prolapse and urinary incontinence. Rev Obstet Gynecol. 2010;3:3-9. 